\title{
Designing on Optical Wireless Communication for 5G Mobile Applications
}

\author{
T.Padmapriya ${ }^{1}$, S.V.Manikanthan ${ }^{2}$ \\ ${ }^{1}$ Managing Director, Melange Academic Research Associates, Puducherry \\ ${ }^{2}$ Director, Melange Academic Research Associates, Puducherry
}

Article History: Received: 10 November 2020; Revised: 12 January 2021; Accepted: 27January 2021; Published online: 05April 2021

\begin{abstract}
The emphasis of this paper is on designing 5G networks infrastructure, offering a better wireless and optical network segment transport scheme with domains for applications for mobile phones. Identify the appropriate set of power grid wireless (optical/optical) technologies) and processing modules needed to facilitate 5G mobile services in an energyefficient a two-stage optimization framework and the premium way is suspected. A multi-objective method in the first iteration is for optimization, where reflects on the element of the transport network strives to jointly reduce the expenditures on investment of the $5 \mathrm{G}$ mobile network. This is done to determine the minimum mobile technologies for wireless and optical power grids. The second stage focuses on the branch of the web server and aims to define effective modules of processing to which $5 \mathrm{G}$ operational systems need to be distributed. The performances of the proposal are investigated using separate clustering algorithms, reasonable traffic statistics choices such as mm-wave and optical passive networks (PONs) for optical grid transport, fixed, and elastic networks in Bristol, UK, via a city-wide topology. To give more advantages to 5G applications, our paper proposed a 5G communication using optical wireless technology.

Keywords: 5G, power grids, optical passive network(PON), mobile technologies, topology, elastic network.
\end{abstract}

\section{Introduction}

5G is the fifth generation standard of technology in broadband cellular network telecommunications, which mobile operators recently implemented nationwide in 2019, and is the projected equivalent to the $4 \mathrm{G}$ networks that connect directly to most established cell phones[1]. According to the GSM Alliance, 5G network can have more than 1.7 billion users worldwide by 2025.[2] 5G networks are digital platforms, including existing predecessors, in which the business zone is divided into the following cells called country towns. All 5G wireless devices in a cell are connected to the internet and telephone network by radio waves by such a local antenna in the cell. The primary advantage of new networks is that they'll have time and increase, faster download speeds[1] and up to 10 gigabits per second (Gbit/s) in aggregate [3]. Due to the extremely increased bandwidth, networks are now supposed not only to serve mobile devices such as existing cellular sensors, but also to be used as general internet service providers for laptops and desktop computers, to compete for ISPs such as cable internet, and to make a new Internet of Things (IoT) and machine-to-machine possible applications. New networks that also need 5Compliance issues wireless devices are not able to produce 4G cell phones.

The greater speed is most often reached by using radio waves of higher frequency than previous cellular networks.[1] However, radio waves of higher frequency have a shorter physical range of its use, requiring smaller regional cells. 5G devices transmit on up to three frequency bands for global presence devices, low, medium, and powerful.A 5G network will consist of networks of up to three cellular organelles, each designed for individual antenna layouts, each with a specific trade-off for download latency vs. distance and coverage area. $5 \mathrm{G}$ mobile phones the low-band $5 \mathrm{G}$ uses $600-850 \mathrm{MHz} 4 \mathrm{G}$ phones with a similar frequency range, bringing throughput slightly higher than 4G: 30-250 megabits per second (Kbit / s] Wireless devices interface to the server at their location through both the highest speed antenna within their range.[4] This same 4G staircase range and coverage area of low-band cell towers. This same $5 \mathrm{G}$ mid-band it uses $2.5-3.7 \mathrm{GHz}$ microwaves, allowing speeds of 100-900 Mbit/s, with a radius of up to many other miles for each cell tower. This is the most generally deployed level of service It should be available by 2020 in most metropolitan areas. There are no lowband implementations for certain nations, making this the minimum rate of the material. At the bottom of the millimeter-wave band, high-band $5 \mathrm{G}$ uses frequencies of 25-39 GHz, but higher frequencies will be used in the future. It also maintains downloads equal to the streaming service in the gigabit per second (Gbit/s) range. A more minimal number of microwaves (mmWave or $\mathrm{mmW}$ ) is, but will need multiple cells. Several types of materials have trouble breathing through them, such as walls and windows. Plans are to integrate these cells only because of their higher cost in dense urban environments and areas, such as sports stadiums and conference halls[55], where large numbers of people cluster. The latter speeds are those produced for 2020 in the standard results, and speeds are expected to increase for the year roll-out.

5G networks are digital cellular networks that divide the network coverage covered by distributors into small geographical areas called cells. On the telephone, binary signals expressing sounds and images are decoded, converted by an analog-to-digital converter, and transmitted as a bitstream. Any 5G wireless devices in a cell 
communicate using radio waves with a local antenna array and a low-power automated transceiver (transmitter and receiver) in the cell, Over the frequency channels determined by the transceiver[6], from a pool of frequencies reused in other cells. Wireless backhaul connectivity or high-bandwidth optical transmission attaches the local antennas to telephone and Internet infrastructure 5G networks are digital cellular networks that divide the available bandwidth covered by distributors into small geographical areas called cells. Analog audio and video signals are modified on the telephone, converted, and broadcast as a bitstream through in an analog-todigital converter. 5G wireless devices in a cell communicate from a pool of frequencies reused in other cells using radio waves with a local antenna array and a low-power automated transceiver (transmitter and receiver) in the cell, over frequency channels issued by the transceiver.

A high-bandwidth optical transmission or backhaul wireless relation connects the local antennas to the telephone network and internet. A mobile device is probably taken aside' directly to the new cell that changes from one cell to another, as in other cell networks. Up to a million devices per city, the block will be covered by $5 \mathrm{G}$, as only up to 100,000 devices per square kilometer[7][8] are provided by 4G. The new 5G wireless devices now have $4 \mathrm{G}$ LTE technology, as $4 \mathrm{G}$ is used by the new networks to initially access the network, as well as in regions where $5 \mathrm{G}$ communication is not possible. The $4 \mathrm{G}$ LTE feature of the current $5 \mathrm{G}$ wireless communication systems will be, as $4 \mathrm{G}$ is used by new networks to initially connect to the mobile, as well as in instances where $5 \mathrm{G}$ networking is not reachable. For greater energy, as well as continue, most network operators use kilometer waves.[9] Millimeter waves have a lower number than microwaves, narrowing the cells to a minimal footprint.

The overall 5G screen goes Beyond the evolution of mobile broadband, the purpose is to create a digital future for an economy in which most industries are disrupted. A 5G catalyst is the payment to startups. Manufacturing industry stakeholders (referred to as vertical industries)new business models and possibilities, and promote them. This, vision establishes the need for mainstream transition from scalable, elastic landscapes, closed and inelastic infrastructures for open networks, necessary to sustain a huge ecosystem. Variety of programs and resources that overlap dynamically. A selection of heterogeneous ecosystems would also be mixed into these ecosystems. Via high-capacity networks, a massive indicator of engineer's produce is interconnected by air interfaces such as 3G, 4G, and Wi-Fi. The long/short radio access network (RAN) research applies productivity to improve accuracy with reduced spectral radio access network (RAN) deployments molecules. But every tactic means better demands in terms of baseband units (BBUs) although this RAN application for load-bearing structures is (D-RAN) architecture already embraced. This is a measure to ensure that one BBU is co-located and desired per radio system in D-RANs, radio units, and BBUs. The treatment of added revenue. The technical and management costs associated with this project, the Cloud Radio Access Networks (C-RANs) specification, have been registered. In C-RAN, remote units (RUs) access points (APs) are very often identified to be affiliated with a central unit (CU) where there would be a BBU pool known as front haul (FH) by highbandwidth transport. Figure 1. shows the schematic diagram of the $5 \mathrm{G}$ network.

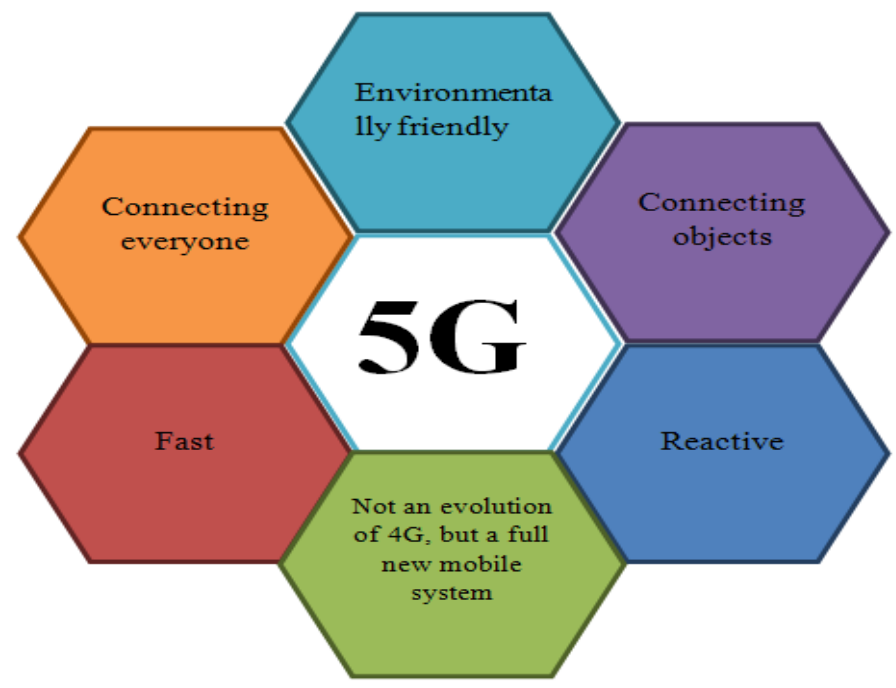

Figure 1.schematic diagram of $5 \mathrm{G}$

Services from FH are fully represented using protocols like the popular interface with public radio (CPRI). Digitized adoption transmission prevents the amount of the signal and requires the transmission to longer-reach delivery, allowing a high amount of solidifying BBU. The C-RAN architecture, therefore, provides better 
performance by BBU sharing. Similarly, the sharing between a set of RUs of a single BBU pool its material increases the needed organizational purposes in deployments through MIMO. These disadvantages, however, come at a cost of increased transport network capacity which can exceed $10 \mathrm{~s} \mathrm{of} \mathrm{Gb/s,} \mathrm{which} \mathrm{is} \mathrm{also} \mathrm{very} \mathrm{poor} \mathrm{in}$ diverse RAN solutions, delay, and very strong (less than $1 \mu \mathrm{s}$ for the FH segment) requirements surrounding compatibility. Including the current optical, transport mechanisms are commonly either passive or passive. Gigabit-capable passive optical network (PON), an optical network (PON), Network (GPON), or 10GE technologies, which are willing to help. These technology options can have capacities of up to 10 Gbps, unable to provide the increased requirements for transport RANs of the next-generation.This same optical wireless network (OWC) is an optical authentication scheme in which visible, infrared (IR) or ultraviolet (UV) light is unguided acts as a gateway.

OWC communication components are largely referred to as visible light communication systems mostly in visible band (390-750 $\mathrm{nm}$ ) (VLC). Light-emitting diodes (LEDs) can also be used by VLC mechanisms and can only be pulsed at very high speeds without damaging effects on the lighting operation and the human eye. In a large variety of applications, also including local wireless networks, personal wireless networks, and vehicular networks, VLC can be used point-to-point OWC implementations, on the other hand, based on space, always known to as Free Space Optical (FSO) systems, are used. These courses typically use laser transmitters, i.e. 10 Gbit/s per wavelength, to provide high data rates, with a cost-effective protocol-transparent reference, and provide a possible answer for the backhaul bottleneck. As a result of recent developments in solid-state optical sources/detectors located within the solar-blind Ultraviolet region $(200-280 \mathrm{~nm})$, there has been a strong number in ultraviolet communication (UVC) as well.

In this so-called deep UV band, solar radiation at the ground level is negligible and this increases the design of photon-counting detectors with vast field-of-view receivers that increase the power obtained with little unwanted background noise. Those other designs are exactly effective for non-line-of-sight outdoor installations to support short-range installations UVCs with low power, such as connected devices and ad-hoc networks. In a variety of communicative applications, OWC modifications can likewise be utilized, outdoor inter-building connection to satellite communications occur from optical inter-dependencies within integrated circuits. OWC can be broken into three various components based on the absorption spectra,

* Ultra-short range: In stacked and cramped multi-chip packages, chip-to-chip messaging

* Short-range: IEEE 802.15.7 standard Wireless Body Area Network(WBAN) and Wireless Personal Area Network (WPAN) applications, underwater communications

* Long-range: inter-building communication, also known as optical communication in free space.

The remaining of this paper is organized as follows.Section 1. highlights the proposed system of mobile applications with optical wireless communication.In section 2, shows the how transmitter and the receiver work in optical wireless communication. In section 3.reveals the results and conclusion of this paper.

\section{Related Works}

The author Al-Kinani, A., Wang, C. X., Zhou, L., \& Zhang, W[10]explained that optical wireless communications (OWCs) are cellular modems systems are using optical carriers in the electromagnetic spectrum of infrared, visible light, or ultraviolet bands. Comprehensive understanding and specific contact prediction behavior and attitude are important in the process of the design and performance of an OWC link measurement. For the OWC device is connected, efficient and accurate channel models are therefore crucial. Firstly, this paper gives a brief overview of that same OWC. OWC channel scenarios and their management currency are also based on consideration of the optical carrier, range, mobility, and network bandwidth. The key elements of the optical channel that regulate the optical channel, by, reliability of the OWC channel are also being investigated. A thorough analysis of the most appropriate campaigns and channel models tackles channel measurement for OWCs, particularly for wireless infrared communications and visible light communications. In terms of amplitude, complexity, and accuracy of computation, OWC channel models are further compared. The survey considers interactive contexts outside, elsewhere, massive underground, and underwater. Finally, the channel measurements and models start providing future research directions of OWCs.

The author Chowdhury, M. Z, et al..,[11] described that the new versus the current fourth-generation handheld device, the system components of the fifth and sixth iterations (5G and 6G respectively) are widely used for solving the Big Data age. The few common nutritional issues related to 5G and 6G telecommunication systems' service quality are star topology, high connectivity, low latency, high availability, low energy demand, high experience quality, and connectivity output. Of course, compared to $5 \mathrm{G}$ communication on these matters, 
6G communication could have several times increased performance. The tactile intertubes Internet of Things (IoT) would also be an integral part of $5 \mathrm{G}$ and beyond (5 GB) communication networks (e.g. $5 \mathrm{G}$ and $6 \mathrm{G}$ ). Furthermore, in implementing the narrow assumptions of heterogeneous traffic and implementing the conditions relating to service security, 5 GB wireless networks will face several challenges identified. Along with many other wireless technologies, optical wireless communication (OWC) A promising candidate for 5 GB communication systems to meet the requirements. This research chapter systematically presents how OWC technologies can be an important opportunity for 5G/6G and Smart appliances, it could be conspicuously used for visible light communication, light consistency, optical camera communication, and free-space optics communication.

The author Kishore, V et al..,[12]explained that optical Wireless Communication (OWC) was being championed by the advent of the Internet of Things (IoT) to meet the insufficient future broadband wireless systems' data needs. However, these OWC systems rely on orthogonal Frequency Division Multiplexing (OFDM) waveform variants that suffer from efficiency, spectral efficiency, and power efficiency. A DC Multiplexing Weighted Optical Universal Frequency Division (DCO-GFDM) is based on burst transmission for OWC systems based on electromagnetic waves (IM) and direct detection (DD) using circular to solve these issues, rotating pulse shaping is combined. Assorted receivers are introduced, utilize Dirichlet pulses, and Root Boosted Cosine (RRC). Compared to DC weighted Optical OFDM (DCO-OFDM) using two generalized LED models under Additive White Gaussian Noise (AWGN) channel (SER) accuracy, the effect of the inevitable non-linearity distortion developed at the Light Emitting Diode (LED) on the Symbol Error Rate is waveform described. The consequence of LED clipping is investigated at different using Monte-Carlo simulations, restraints concerning DC-bias factor, transmission power and LED dynamic range. Results show that even under LED nonlinear distortion, SER potential is matched to DCO-OFDM, but with more precision in waveform formation, high operational efficiency, and up to $20 \mathrm{~dB}$ reduction in outbound carrier power efficiency.

The author Nguyen, H, et al..,[13] explained the whole orthogonal multiplexing of OFDM is a digital multicarrier take-input that is used as an important definition through inter-symbol interference enhanced by a multipath channel wired and wireless broadband communication. The OFDM waveform is well-known and commonly applied in light fidelity, so the use of such equipment for optical camera communication (OCC) is novel. The OFDM OCC one-dimensional platform is discussed in this section. A rolling-shutter effect has been used by the modulation scheme based on OFDM To design an economic study emphasis on theoretical and experimental validation with various technical aspects, such as cost of innovation and theoretical expenses.

The author Mesleh, R., \& Ayat, A. O[14] described that provides and examines a network communication system including Free Space Optical (FSO). In an attempt to promote a Bragg cell at the Bragg angle, an incident laser beam is aligned. At the same time, the data input bits to be transmitted are influenced by the RF signal frequency, which will in turn only establishes the acoustic wave propagating in the Bragg cell. As such, based on acoustic wave frequency, information carried multiple diffracted beams can be developed in the Bragg cell. In a constellation diagram, the different current item variant signals are used and are used to communicate data to the destination from the source. It assesses the diffracted beams of the variant where added to the receiver with so many other photodetectors. In the receipt of information bits, and accurate receiver might study the social angles and intensities of the transmitted beams. The wireless communication system's average bit error probability is obtained and analytically validated by Monte Carlo simulations. The analysis, in reference, is based on the platform and interpretation of the channel. In comparison to conventional state-of-the-art FSO systems, observed results demonstrate vast improvements in the proposed algorithm.

The author Janevski, T.[15] explained that the 3Cellular network networks are on the ground today, actually offering and non-real-time services including IP access. On the other contrary, there are many common wireless technologies, with the most important ad hoc Wireless Personal Area Networks are 802.11 Wireless Local Area Networks (WLAN) and 802.16 Wireless Metropolitan Area Networks (WMAN) as well as digital public television programming wireless networks. Well, there is already much awareness of the standards of $4 \mathrm{G}$ and it is almost likely that under an up a time, $4 \mathrm{G}$ will include certain standards, similar to $3 \mathrm{G}$, but included from the inception through cellular IEEE 802.xx networks. The purpose of this article is to explain the idea of 5G service providers (fifth generation), which is seen as a concept of the viewer but not as a concept of the telecommunications operator as seen in $3 \mathrm{G}$ or consumer experience for $4 \mathrm{G}$.

The computer application is on top of anything in the proposed definition. The 5G terminals might have radios and modulation techniques for development tools, as well as new existing infrastructure defects that can be downloaded from the internet after they worked. Installation of mobile terminals is seen as a hallmark of mobile 5G networks. Terminals are pushed to provide both that between wireless devices and varying flows of 
different technologies should be able to be integrated by the terminal. The preserving user mobility for each network will be, although the terminal will make the final preference between the various wireless providers of a for a specific route, network. The paper also established a good internet phone concept in which all phones can choose and optimize the best links over selected constraints during a single end-to-end connection. The idea in this paper is a major improvement like mobile networking higher than traditional $3 \mathrm{G}$ and near-soon $4 \mathrm{G}$ consumer communications, and this idea is here pointed to as $5 \mathrm{G}$.

The author Le, L. B., Lau, V., Jorswieck, E., Dao, N. D., Haghighat, A., Kim, D. I., \& Le-Ngoc, T[16] explained that in the past few years, both academia and industry have been strongly interested in studying on 5G applications for mobile users. There is some controversy about several wireless enabling technologies, it has also been kept in mind to achieve these organizational plans of $5 \mathrm{G}$ wireless connections (e.g. data rate, network connectivity, delay). It was, however, very clear that no single enabling technology should arise so could withstand all the similar and even contrasting 5G instances. In general, the future $5 \mathrm{G}$ will need several big advances to re-engineer deeper components of the overall network architecture and algorithms and increase new degrees of device transparency.

In particular, other possible waveform candidates may need to be identified Develop ambitious projects to meet $5 \mathrm{G}$ rate and frequency conditions, including network densification, large-scale widespread availability, and multiple-input multiple-output (MIMO) exploitation, which can resolve the limitations of multiple access orthogonal frequency (OFDM) wavelengths used during the current client limitations of network harmonics. Also, computer networking domain design tools are expected to play out along with the software-defined networking, virtualization, and cloud computing out again in defining the more mobile, flexible, and productive 5G network architecture major position. This paper aims to address the main 5G mobile communications technology and to tackle their potential and the obstacles of open research. Begin to understand how scientific papers in our special issue encourage the creation of these nascent $5 \mathrm{G}$ technologies.

The author Ding, Z., et al..,[17] explained that recently, non-orthogonal multiple access (NOMA) was mentioned for 3GPP LTE as the current head of the multiple access family and is anticipated to be an integral part of 5G mobile networks. NOMA's best attraction is to handle large users with different power levels a systemic MA is generated at the same time/frequency/code, which results in an extended wait over spectral quality. The report provides a critical description of this newly emerging technology, from its combination with MIMO to NOMA's cooperative technologies, as well as the interface between NOMA and cognitive radio. This paper describes the state-of-the-art establishment and the world taking part in the adoption of NOMA on LTE and $5 \mathrm{G}$ networks.

The author Mamode, M. I. S, et al..,[18] described that in terms of connectivity, mobility, speed, and latency, $5 \mathrm{G}$ mobile communication technologies are guaranteed to offer great changes to traditional systems. They can make automation realistic by improving access and business models in many businesses and vertical markets. In terms of scheduling contributing to the E-UTRAN 5G, most changes have happened about the scheduling (Evolved-Universal Terrestrial Radio Access Network). The aim of this exercise is two-fold. First, including some new features added by $5 \mathrm{G}$ by and, an analysis of the $5 \mathrm{G}$ network architecture, scheduling process, and some existing scheduling algorithms is presented. Secondly, innovative scheduling approaches are studied and directions are suggested for possible work and changes made.

\section{Proposed Work}

The domain and radiation/detection technologies the indoor or outdoor transmitters and receiversatmosphere specify the geometry of a wireless communication scenario. Environment, with the objects' certain reflection properties in the setup. Based on the thermal transfer trajectory that the transmitter radiates and the receiver detects, two general arrangements for ties arise, i.e. communications from LOS and NLOS. Also, it is possible to deploy a cellular network to optimize coverage and capacity over the setup area including its OWC. This list comprises of building blocks. The front-ends of the transmitter and receiver and the general communications procedures are established for deployment are discussed. Digital-to-analog converter (DAC) signal processor (DSP) to suit the signal processor (DSP) digital information bits are amplified and shaped into a traditional currency. demonstrating signal.

An optical emitter, i.e. an LED or an array of LEDs, drives the current. Here the current signal carrying information is processed into optical pressure. The optical solution to further influence the transmitted signal, signal may be passed via an optical system beam. Here it is important to use an optical amplifier lens, a collimator, or a nozzle to situate or broaden the projector. The optical signal is then communicated by the optical signal channel wireless. The products in the objects retain a portion of the optical energy of the world, and in a 
diffuse or specular shape, the rest is reflected. And LOS and at the receiver, NLOS signal components arrive. To sort, an optical regulator can be extended to part of the optical spectrum of interest. However, the optical philter greatly diminishes ambient light intervention.

Finally, the optical signal is updated by a system of optical systems, e.g. collimator lenses, for suggested solutions and Intrusive light convergence for identification and detection. The photodetector, i.e. a detector for PD or PD. Initially, the optical signal, an array of PDs, is converted to electric current. To use a trans-impedance amplifier, the current signal is electronically pre-amplified to (TIA). DSP with an analog-to-digital converter (ADC) can be used to transform. The date signal into a digital signal and the signal demodulation bits. Likewise, based on the connections are defined as ratio forcefulness between the transmitter and the receiver, as nondirected and directed. Even though oriented LOS links have quite a high receiver irradiation intensity and a microchannel coherence bandwidth, which may not be ideal where only high user mobility is high is demanded and the interaction can easily be intercepted or interrupted.

As a non-directed NLOS messaging, on the other hand, offers a non-directed NLOS communication, lower irradiation concentration, and a narrower bandwidth of channel coherence at the receiver, the NLOS scenario serves user mobility better and is slightly more stable. Exploiting the reflection properties of the room materials, an NLOS coordination setup, irradiation intensity can be increased at the receiver diffusion by spot. This is incredibly valuable for infrared (IR) spectrum partnerships, where the reflectivity of the object in addition to its main than that of visible light. Since the primary visible light contact interface is (VLC) links are illumination, a uniform distribution of light over the room's topology is needed. After all, it is shown that it is possible to visualize the irradiation strength at the receiver further supported by spotlighting. Clear and unique optics have been carried out.

Under various based on direct measurements or simulations, spatial conditions and optical configurations of ray-tracing wireless networks. It's been has shown that its RMS delay can well represent the optical wireless channel. After all, it is shown that it is possible to visualize the irradiation strength at the receiver. Further supported by spotlighting. Clear and unique optics have been carried out.Under various based on direct measurements or measurements, spatial conditions and optical configurations simulations of ray-tracing wireless networks. It's been has shown that its RMS delay can well represent the optical wireless channel. Up to $13 \mathrm{~ns}$ of RMS delay and up to $100 \mathrm{~ns}$ of total delay duration LOS and NLOS associations have been confirmed, while optical path losses of up to $80 \mathrm{~dB}$ can be established. In an indoor framework, be felt.

\section{Line-of-sight communication}

There is a real plan between the radiation pattern without obstruction and spatial alignment of the transmitter and the receiver detection pattern. The light propagation aspect is referred to as the portion of the light that is radiated by a transmitter that arrives directly from the inside of the receiver's field of view (FOV).

\section{Non-line-of-sight communication}

Two common NLOS communication reference configurations are discussed in the literature: directed NLOS and (diffuse) non-directed NLOS. Electronic interference, e.g. a room wall, the transmitter, and the transmitter With either a single reflection on the ceiling or another wall, the receiver communicates. In NLOS communication, after one or one the signal arrives at the receiver. The objects in the room can bounce off too many times. Trying to distinguish the two NLOS conditions. By the transmitter's directivity. In the first example, NLOS, the transmitter, directed. It has a very narrow character of radiation, spreading the light on a specified instant on the surface that operates like a new transmitter. It conveys the light to the receiver based on its characteristic of reflection. This results in a dimension of single-reflection with strong light energy.

The NLOS directed scenario is close to the proliferation of spots. In the entire first half, In the case of nondirected NLOS, the transmitter has a large radiation function that displaces oxygen. The reflecting surface is a big portion. In a closed room, the radiated environment is likely to be after one or more reflections on the surfaces, the light will arrive at the receiver, creating.The NLOS part of the propagation of light is referred to as the portion of the energy transmitted by the transmitter. After one or more reflections on the subjects in the geometry of the receiver arrives at the receiver in the bed.

\section{Optical front-ends}


The transmitter and receiver optical front-ends contain optical as well as receiver technology. Once the respective electrical-to-optical $(\mathrm{E} / \mathrm{O})$ and optical-to-electrical $(\mathrm{O} / \mathrm{E})$ modifications take place as electrical components, In the following, the alloy steel of the transmitter and the transmitter are seen. Key parameters related to the development of systems company needs for the characteristics of radiation and detection are discussed, including, the optical spectral response, the electrical modulation of the transmitter and the receiver bandwidth, as well as noise from its receiver.

\section{Transmitter}

The transmitter's best characteristics include the pattern of radiation, the optical spectral Solution, the functionality of the E/O switch, and the bandwidth of electrical modulation.A single LED's radiation characteristic is usually described using a schematic Lambertian pattern of radiation. An LED's FOV is defined as the angle. The radiation pattern between the points where the directivity is reduced to five percent. The FOV is traditionally described by the semi-angle between the directions of the FOV. Maximum directivity and directivity of $50 \%, \mathrm{FOV}, \mathrm{T}_{\mathrm{xx}}$. In a given case, multiple LEDs may provide a higher radiant brightness. Place, when constructively reconciling their radiation patterns, besides which, some by co-locating LEDs in a nonplanar fashion, radiation pattern can be obtained. Consequently, it is essential to establish omnidirectional nonplanar transmitters to act as access points (APs) in a network of cellulars. In addition to the radiated optical power of the total output, the spectral response of the optical power is the transmitter can be used in the configuration of OWC cellular networks of high capacity.

The APs in the different tissues can be tuned to different optical hub wavelengths for this purpose, providing non-interfering bandwidths for electrical modulation. Higher wavelength reuse factors in OWC The total machine efficiency may be used for a perceivable reduction, since The electric spectrum that the OWC system uses, e.g. up to a few hundred MHz. It is significantly lower than the amount with state-of-the-art off-the-shelf components, in the optical spectrum, electrical bandwidth available, e.g. approximately $670 \mathrm{THz}$. As a result, the bandwidth of electrical modulation in the order of a few tens of $\mathrm{MHz}$ is used. It is dramatically lower at a given optical power level than the distance between two frequencies for the optical core. Therefore very high reuse variables of wavelength can be incorporated into innovations in optical and electrical filtering. The below figure 2. Shows the block diagram of optical wireless communication.

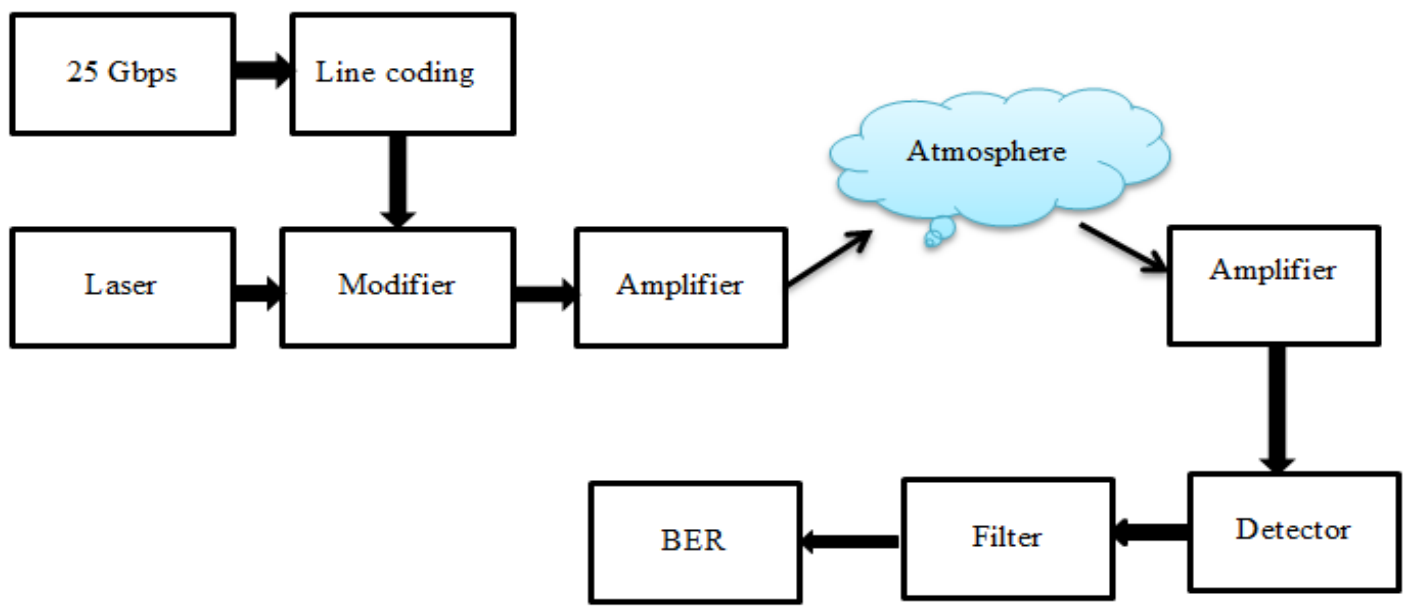

Figure 2. Optical wireless communication

\section{Receiver}

The receiver's major elements include the pattern of detection, the optical spectral resolve, the functionality of the $\mathrm{O} / \mathrm{E}$ conversion, the bandwidth of electrical modulation, and the figure of noise.In general, the detection characteristic of a single PD is established using the means of a pattern of Lambertian detection. Similar to something like the LED, A PD's FOV is defined as the angle of the detection pattern between the points, where the directivity is reduced to $50 \%$. Nevertheless, the receiver's FOV can be a system of attached optical elements that has further reinforced or confined it. For reference, it is possible to use an optical concentrator in the form of collimator lenses to extend the detection software and strengthen the transmit power receiver.Accordingly, the additional FOV parameter is commonly utilized to the optical elements attached engineering the overall 
detection element of the PD to the detection pattern of the PD a recipient. The receiver's optical spectral response influences the response. PD and the spectral response including its optical filter.

A PD's spectral response is generally described by the responsivity parameter i.e. the optical power transfer factor to the optical spectral distance, the electrical current. There is a linear conversion over a considerably high operating range between the receiver's input optical power and output, electrical power. Signal clipping but with PD saturation can be avoided using the automatic saturation formulations used to gain influence (AGC). The receiver's electrical modulation bandwidth is dictated by the electrical bandwidths of the TIA and the PD. The receiver's electrical bandwidth is substantially larger than the transmitter's electrical bandwidth. Bandwidths over capacity it has been documented about $100 \mathrm{MHz}$. The at the receiver in OWC systems, ambient light creates substantial shot noise. Thermal noise, also, increases in the receiver frontend due to the electronic pre-amplifier, i.e. the TIA.

\section{Optical wireless channel}

Has been shown that the linear time-invariant optical wireless channel is, memoryless one. Application with a finite time impulse response. Modulation of magnitude and the most suitable model for OWC is direct detection (IM/DD). Here the amplitude of the current image transmitted is proportional to the optical incident integral. Regulate over the highway patrol. As the area of a PD is millions of spheres, in general, there is a rich spatial diversity of wavelengths. There is, as a result, no rapid fading in OWC, however in the form of shadowing, just rapid fading. The following are successfully make for the OWC, including the collaboration agency theory in the presence of OWC, is discussed. Receiver noise, loss, and delay spread models for the beam axis, as well as techniques at the receiver, for data compression.

\section{Channel model}

The following continuous-time model for a noisy active area can be explained by OWC.

$$
\mathrm{a}=\mathrm{k} * \mathrm{~F}(\mathrm{x})+\mathrm{g}
$$

where a(t) Reinforces the fragmented replica of the received transmitted signal, $x(t)$ uncovered by the nonlinear distortion structure, which, the non-linear function of distortion is introduced to $F((x) t)$, the front-end transmitter. With the channel impulse, the non-linearly distorted transmitted information is modulated. Respond, $\mathrm{k}(\mathrm{t})$, and is manipulated by the receiver by AWGN, w(t), Here, linear denotes nervous breakdown. convolution. The OWC relation structured model in the time domain is illustrated. As the policy of the OWC is followed using a DSP, the following become adopted are implemented. Then by using an opposite discrete model for a noisy communication channel description.

\section{Ray-tracing for the prediction of signal and interference}

Modeling the propagation in an indoor environment of an optical wireless signal is a stressful job. The highly conductive qualities of the surfaces on the inside of the structure. It affects signal fuel economy. In particular, any modification, the transmitter and/or receiver's AZ, EL, or FOV determines the channel interfaces.Additionally, the properties of the channel also vary in acceleration, blockage, and shadowing. Cochannel-interference-is a configurement of hexagonal columns and reuse of inputs 3. (CCI)-limited for small cell radii and noise- restricted but not limited towards large cell radii, for SIR spatial distribution example is given. Peer-to-peer indoors with the client/server wireless topologies have been explored, but it is expected that a noiselimited network. Fortunately, because with the projected higher data the wireless network is impaired by interference in the density of an aircraft cabin. SIR maps, hence in this section to calculate the lowest, reuse variables are measured for specific scales.

Achievable SIR at the edges of layers that series the highest CCII. The major challenge in the prediction of the complexity of a simulation of global electricity, including one with a model of geometric setup, material reflection properties, radiation pattern classification, is related to path loss. The transmitters, and the family pattern of detection. Measurements and sizes tests suggest that optical path loss varies approximately $50 \mathrm{~dB}$ to $50 \mathrm{~dB}$. And $80 \mathrm{~dB}$ for diffuse channels with single reflection, unshadowed or shadowedamount equal the results demonstrated that the distribution of loss of direction along the direction is smooth, to accurately estimate the intermediate values, straightforward curve-fitting can be used. What the existing practices normally lack, however an automated way to measure the loss comprehensively is off the path at any situation possible in a chosen room or setup. As a distance function, an optical wireless path loss statistical system is needed in various 
indoor conditions, such as an aircraft cabin. Such, a flexible model would permit the position and parameters of the environment to be controlled. The transmitters, AZ, EL, and FOV, for starters, and receivers. In particular, SIR maps over many sizes of Tx and Rx and common reuse factors for all of these electromagnetic spectrum configurations. This same world is still an unanswered problem.

\section{Estimation of line-of-sight path loss and shadowing}

The irradiance distribution is simulated three percent stochastic security criterion in the setup with the explicit numerical optical communication scenario to an MCRT simulation stopping criterion. The exponent of track loss, $\Omega$, and the usual shadowing deviation when deciding the factor, $\sigma$, can be decided by path loss along the four priority routes in the aircraft cabin. Since the PD photosensitive region expression is indeed an honest argument in the equation, the path loss curve only enhances the lives without affecting its slope or the absorbance deviation due to shadowing possibilities. The photosensitive PD layer is used uniquely to approximate the constant values in the 4-direction equation. The distance through path 2 to transmitter 1 is 0.42 $\mathrm{m}$, although this distance through path 2 to transmitter 1 is $0.42 \mathrm{~m}$. The sidewall is $0.18 \mathrm{~m}$ from the lane. Similarly, to measure the loss of the road. The exponent only receives the information in the proper direction" to access the receivers. The conditions mentioned multipath signal element is irradiated along the route by the receivers. The receivers thus receive along the path to measure the exponent of path loss. The signal that determines the signal that determines the reception directions for "back "front" left-right" reception directions. The receivers are selected in the individual exposures to estimate the shadowing variable. From overlapping rings around the transmitter middle 1 . To estimate the shadowing variable."

\section{Signal-to-interference ratio maps}

The previously discussed division of the cabin of the aircraft into nine increased the level it clearer to understand the distribution of the SIR for different factors of wavelength reuse. It, in the simulation, the interference is considered appropriate only by co-channel signals and signals corresponding signals. It is difficult to prevent channel interference (ACI) by-products containing LED-PD pairs. Two LED-PD pairs, generated by semiconductors, is being used as an example.The TSFF5210 LED and TESP5700 PD have peak spectral emission and responsivity at peak spectral emission and reaction. The VSMB2020X01 LED and the PD BPW41N LED have spectral peaks. At $940 \mathrm{~nm}$ in emission/sensitivity. More about peak wavelengths, their 3$\mathrm{dB}$ bandwidths do ACI should therefore be considered to be sufficiently low and not overlap, since the shadow, since the light it is helpful to simulate the distribution separately for each of the nine transmitters, according to the wavelength, to recognize the interfering cells for every intended cell division and mission.

The placement of the transmitters in the horizontal plane in the downtown landscape in which they should be mounted, transmitters is convenient for receiver locations. Yet, considering the user versatility and the diverse use cases make it virtually impossible for a flexible transmitter to be operating. In all future elevation directions, to radiate the same power. Therefore the SIR distributions through the cabin at economically diverse levels provide an important insight into SIR allocation. If the transmitters do not point in the direction of the real SIR, the status of a user is the SIR implementable, i.e. the SIR when the user occurs well beyond the boundary.

\section{Results}

The proposed technique of optimization is evaluated using the topology of the $5 \mathrm{G}$ network involving elements of electronic, optical, and DC. This topology comprises a zone of $10 \mathrm{x} 10 \mathrm{~km} 50$ RUs are dispersed over it. End-users, therefore, acted as licensed here and by the RUs. This implies the traffic that several cloud providers can process. The usefulness of the software both for transport network as defined by the gross CAPEX and OPEX. In the numerical results, it was concluded that speed rate is related to carbon intake and was related to monetary values by multiplying R.u/kWh by 0.02 . It is shown that when the maximum CAPEX and OPEX are still backhauled by RUs, Raman spectroscopy is reduced elevation. It was because of the relatively high use of resources. Levels of mm-wave connectivity (lowest penetration of WDM) and as required, This, also, is due to an OPEX improvement. On the other hand, the decrease in WDM-PON penetration reduces the benefit of the energy-efficient device to that of the PONs. The WDM-PON backhaul, however, reaches a specific number of RUs, corresponding to rises in cumulative OPEX and CAPEX. This is credited to an appropriate textile that is costs with as such, optical trenching also due to the reduction of CAPEX associated through CAPEX. The impact of the penetration of bit error rate and speed of 5G mobile applications is shown in below figure 3 . 


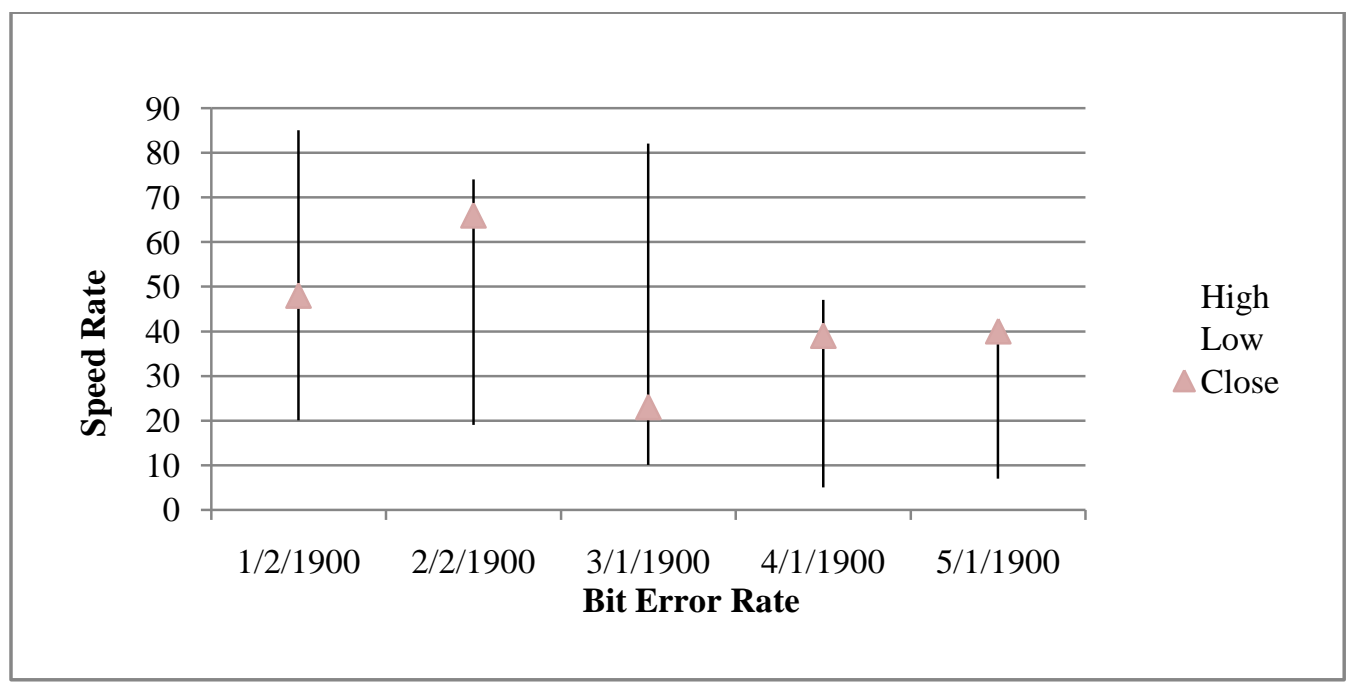

Figure 3. Bit Error Rate and Speed Rate of optical wireless communication for $5 \mathrm{G}$ mobile applications

And is shown to be high for bit error rate values, enhanced split options values. It is preferable (light CPRI flows), as the operational flows the expense of the microwave transport of heavy there is low-density CPRI flows. On the other hand, an expansion in the penetration of bit error rate corresponds to an acceleration of the converged network infrastructure of the transport network, allowing for choosing bandwidth the calls for split resources

\section{Conclusion}

Design and engineering in the latest studies system would make it difficult to evaluate the minimum working conditions are presented and introduced in a converged 5G network. The proposed network consists of point-topoint microwave connections and passive and active IOT application technologies for the utility of BH and FH services. In order, a process for multi-stage optimization has been studied to enhance the architecture proposed. The analysis of quantities, the use of rational traffic statistics, and the $5 \mathrm{G}$ topology of the capital in Bristol, UK, have shown that the viable preference of power grid technologies available in the capital can be shown field of transport improved multi-technology infrastructure and allocation of individual BBU functions to specific programming modules currently utilizing reaching better fuel economy. The dynamics of an OWC setup being discussed, including the front-end structure, the telecommunication geometry, and the resulting wireless optical channel. The ordinary building blocks of the transmitter and the receiver were tackled. Also, LOS and NLOS contact possibilities have been developed. The irradiance at the receiver and the coverage can be combined to strengthen theindoor IR and VLC settings given.

\section{References}

1. De Looper, Christian (March 27, 2020). "What is 5G? The next-generation network explained". Digital Trends. Retrieved April 25, 2020.

2. "Positive 5G Outlook Post COVID-19: What Does It Mean for Avid Gamers?". Forest Interactive. Retrieved November 13, 2020

3. Hoffman, Chris (January 7, 2019). "What is 5G, and how fast will it be?". How-To Geek website. HowTo Geek LLC. Archived from the original on January 24, 2019. Retrieved January 23, 2019.

4. M. Sauer, A. Kobyakov, and J. George, "Radio over fibre for picocellular network architectures," J. Lightwave Technol., vol. 25, no. 11, pp. 3301-3320, 2007.

5. "Microwave and millimetre wave for 5G transport," ETSI White Paper 25, Feb. 2018, https://www.etsi.org/images/ files/ETSIWhitePapers/etsi_wp25_mwt_and_5g_FINAL.pdf.

6. Nokia, "Nokia accelerates centralized RAN deployment with expanded mobile fronthaul solution \#MWC16," Nokia Press Release, Feb. 2016.

7. Shatrughan Singh (March 16, 2018). "Eight Reasons Why 5G Is Better Than 4G". Altran. Archived from the original on May 25, 2019. Retrieved May 25, 2019.

8. Forum, C. L. X. (June 13, 2019). "1 Million IoT Devices per Square Km - Are We Ready for the 5G Transformation?". Medium. Archived from the original on July 12, 2019. Retrieved July 12, 2019.

9. Rappaport, T.S.; Sun, Shu; Mayzus, R.; Zhao, Hang; Azar, Y.; Wang, K.; Wong, G.N.; Schulz, J.K.; Samimi, M. (January 1, 2013). "Millimeter Wave Mobile Communications for 5G Cellular: It Will Work!". IEEE Access. 1: 335-349. doi:10.1109/ACCESS.2013.2260813. ISSN 2169-3536 
10. Al-Kinani, A., Wang, C. X., Zhou, L., \& Zhang, W. (2018). Optical wireless communication channel measurements and models. IEEE Communications Surveys \& Tutorials, 20(3), 1939-1962.

11. Chowdhury, M. Z., Shahjalal, M., Hasan, M., \& Jang, Y. M. (2019). The Role of Optical Wireless Communication Technologies in 5G/6G and IoT Solutions: Prospects, Directions, and Challenges. Applied Sciences, 9(20), 4367.

12. Kishore, V., \& Mani, V. V. (2019). An LED modelled GFDM for optical wireless communications. AEU-International Journal of Electronics and Communications, 101, 54-61.

13. Nguyen, H., Thieu, M. D., Nguyen, T., \& Jang, Y. M. (2019). Rolling OFDM for image sensor based optical wireless communication. IEEE Photonics Journal, 11(4), 1-17.

14. Mesleh, R., \& Ayat, A. O. (2018). Acousto-optical modulators for free space optical wireless communication systems. Journal of Optical Communications and Networking, 10(5), 515-522.

15. Janevski, T. (2009, January). 5G mobile phone concept. In 2009 6th IEEE Consumer Communications and Networking Conference (pp. 1-2). IEEE.

16. Le, L. B., Lau, V., Jorswieck, E., Dao, N. D., Haghighat, A., Kim, D. I., \& Le-Ngoc, T. (2015). Enabling $5 \mathrm{G}$ mobile wireless technologies.

17. Ding, Z., Liu, Y., Choi, J., Sun, Q., Elkashlan, M., Chih-Lin, I., \& Poor, H. V. (2017). Application of non-orthogonal multiple access in LTE and 5G networks. IEEE Communications Magazine, 55(2), 185 191.

18. Mamode, M. I. S., \&Fowdur, T. P. (2020). Survey of Scheduling Schemes in 5G Mobile Communication Systems. Journal of Electrical Engineering, Electronics, Control and Computer Science, 6(2), 21-30 